\title{
Resource Allocation for Relay-Aided Cooperative Systems Based on Multi-Objective Optimization
}

\author{
Runze Wu, Jiajia Zhu, Hailin Hu, Yanhua He, and Liangrui Tang \\ State Key Laboratory of Alternate Electrical Power System with Renewable Energy Sources, North China Electric \\ Power University \\ Beijing 102206, China \\ [e-mail: 15811446189@126.com] \\ *Corresponding author: Jiajia Zhu
}

Received October 13, 2017; accepted January 2, 2018; published May 31, 2018

\begin{abstract}
This paper studies resource allocation schemes for the relay-aided cooperative system consisting of multiple source-destination pairs and decode-forward (DF) relays. Specially, relaying selection, multisubcarrier pairing and assignment, and power allocation are investigated jointly. We consider a combinatorial optimization problem on quality of experience (QoE) and energy consumption based on relay-aided cooperative system. For providing better QoE and lower energy consumption we formulate a multi-objective optimization problem to maximize the total mean opinion score (MOS) value and minimize the total power consumption. To this end, we employ the nondominated sorting genetic algorithm version II (NSGA-II) and obtain sets of Pareto optimal solutions. Specially, two formulas are devised for the optimal solutions of the multi-objective optimization problems with and without a service priority constraint. Moreover, simulation results show that the proposed schemes are superior to the existing ones.
\end{abstract}

Keywords: Resource allocation; relays; multi-objective optimization; nondominated sorting genetic algorithm version II (NSGA-II)

This work was supported in part by the National Natural Science Foundation of China (Grant No.: 51507063) and the Fundamental Research Funds for the Central Universities 2017XS008. 


\section{Introduction}

Relaying has been widely recognized as a promising technique for its ability to enhance the coverage area, transmission reliability and system throughput[1]-[4] . Recently, there is increasig interest in relay networks with multiple source-destination pairs, referred to as multi-user relay networks[5]. Typical multi-user relay networks include heterogeneous relay networks[6], ad hoc, sensor and mesh networks[5]. Works on resource allocation in multi-user relay networks are widely researched. They all devote to answering to the following questions: In a multi-user relay network, which relay node should act as the best relay? Which subcarrier can be shared among multiple source-destination pairs? How to minimize power consumption? Clearly, the answers to these questions depend on the objectives of resource allocation.

\subsection{Related Work}

In the past several years, resource allocation in multi-user relay networks is mainly studied with three objectives: maximizing capacity, energy efficiency(EE) and users' utility. In [6], authors investigate the maximizing capacity resource allocation for backhaul and access links jointly when the bottleneck problem is taken into considration. In [7], authors reconsider the joint hybrid relay scheme and resource allocation for achieving network capacity maximization. Unlike the above resource allocation, authors in [8,9] adopt EE as a new performance metric for designing resource allocation. In [8], authors considered network-wise $\mathrm{EE}$, which is defined as the sum rate over the total power consumption in the overall network. However, in [9], authors investigate the individual EE of each user pair, which is defined as the rate over the power consumption of each user pair. Similar to [8,9], authors in [10] investigate a low-complexity power allocation strategy to minimize sum-source-power for multi-user single-AF-relay networks. Moreover, authors in [11] present a general framework to analyze the tradeoff between the two performance metrics: spectral efficiency (SE) and EE, where resource allocation problem is formulated as a multi-objective optimization problem. Here, SE is essentially the metric capacity. Utility-based resource allocation problem for multi-user relay networks have been investigated in [12] and [13]. In [12], authors use the utility function to describe the degree of user satisfactions, where the resource allocation problem is formulated as a dynamic optimization problem with the aim to maximize the average utility of all users. Authors in [13] consider an energy-aware uitility function , which is defined as the difference between the total income described by capacity and the total energy consumption cost, where the resource allocation problem is formulated as a distributed optimization problem with the aim to maximize the total utility of all users.

Recently, the user-centric [14] concept attracts much attention of researchers. However, in the above mentioned works, there is a main drawbacks that the uses' QoE has not been considered in multi-user relay networks. Fortunately, the QoE-based resource allocation in hetereogeneous networks has been studied widely [14]-[21]. In general, there are two models for evaluating QoE, i.e, MOS and utility functions [14]. MOS [15]-[17] has a score from 1 to 4.5 and reflects the level of user satisfaction. In [15], authors investigate QoE - driven resource allocation, where a multi-objective optimization method is applied to miximaze each user's MOS value. In [16], a QoE-oriented resource allocation problem is formulated as an optimization task to maximazine the overall users' MOS value. In [17], users' MOS value and energy consumption are jointly optimized for achieving significant QoE levels and higher energy efficiency of users. In [18], authors define a utility function to estimate the satisfaction, 
where the resource allocation is analyzed with the objective of maximizing the users' satisfaction across the network. In [19] , authors also define a novel QoE index as the utility function, where the resource allocation is investigated for maximizing QoE while ensuring fairness among users. Furthermore, authors in [20] investigate the combinatorial optimization problem of QoE utility and energy aware in small cell networks, where the resource allocation consists of power selection, load management and channel allocation. In [21], a QoE-based multichannel allocation problem is solved by a joint matching-coalitional game theoretical, where authors considers users' QoE utility and multichannel allocation jointly.

Motivted by the above work, this paper takes two more questions into consideration: In a multi-user relay network, i) What subcarrier pairing strategy should be used? i.e., a common single channel pairing or a special multichannel allocation, similar to [21]. ii) Which metric shoud be selected to evalute users' experience? i.e., three common metrics(capacity, EE and users' utility) or a specific QoE metric.

\subsection{Contributions}

In this paper, we investigate the combinatorial problem of QoE and energy consumption in the relay-aided cooperative system, i.e., in a certain relay network, where the resource allocation consists of relaying selection, multisubcarrier pairing and assignment, and power allocation. This problem is formulated as an optimization task to achieve significant QoE levels and higher energy efficiency of users.

First, a multi-objective optimization framework is proposed with the two objectives of maximizing the total MOS value and minimizing the total power consumption. Then, we employ the NSGA-II to solve this problem and gain the sets of Pareto optimal solutions. Thereafter, two formulas are devised for the optimal solutions of the multi-objective optimization problems with and without a service priority[22,23] constraint. In this way, we can respectively gain the corresponding resource allocation scheme. Finally, to quantify the performance between MOS value and power consumption, the metric of MOS value per Joule is developed. Simulations show that the proposed schemes based on multi-objective optimization can always achieve a better performance in terms of the total MOS value and the metric of MOS value per Joule with lower power consumption by comparisons with the existing works[10] and [12].

The rest of this paper is organized as follows. In Section 2, the system model and problem formulation are introduced. In Section 3, we describe the resource allocation schemes based on multi-objective optimization. Given the multi-objective model, Section 4 presents the simulation results of the resource allocation schemes for the relay-aided cooperative system. Finally, we draw the conclusions in Section 5.

\section{System Model and Problem Formulation}

\subsection{System Model}

In this paper, we consider a relay-aided cooperative system with $M$ source-destination pairs, denoted by $\left(S_{m}, D_{m}\right), m \in\{1,2, \ldots, M\}$ and $L$ relays with DF scheme, denoted by $l, l \in\{1,2, \ldots, L\}$, which is shown in Fig. 1. Similar to the literature [11], we assume that all the relays work in half-duplex mode, which means they cannot transmit and receive signals simultaneously. It is also assumed that channel fading is composed by large-scale fading (path loss) and small-scale fading (frequency-selective Rayleigh fading). In the Fig. 1, each source-destination pair 
exchanges information through one selected relay and the direct link between each source-destination pair is ignored. In order to complete physical transmission, the links from the sources to relays and from relays to the destinations need two phases.

In the first phase, the source $S_{m}$ transmits its signals and the relay $l$ receives them. Define the subcarrier as the basic frequency resource element, and let there be $N$ available subcarriers that can be shared in each phase. Assume that the total bandwidth for $N$ subcarriers is $B$, and the bandwidth for each subcarrier is then $B / N$. For presentation, denote the subcarriers set as $\mathcal{N}$, i.e., $\mathcal{N}=\{1,2, \ldots, N\}$. It is also assumed that the source $S_{m}$ transmits its signals with power $p_{S_{m}, l}^{N_{m}}$ on the subcarriers set $N_{m}$, i.e., $N_{m} \subset \mathcal{N},\left|N_{m}\right|>1$. Furthermore, let $p_{S_{m}, l}^{n_{1}}$ denote the transmit power on the subcarrier $n_{1}$, i.e., $n_{1} \in N_{m}$ and let $p_{s_{m}, l}^{N_{m}}=\sum_{N_{m}} p_{S_{m}, l}^{n_{1}}$. Let $g_{S_{m}, l}^{N_{m}}$ as the channel gain on the subcarriers set $N_{m}$ from the source $S_{m}$ to the relay $l$ and $g_{S_{m}, l}^{n_{1}}$ as the channel gain on the subcarrier $n_{1}$. Especially, let $g_{S_{m}, l}^{N_{m}}=\sum_{N_{m}} g_{S_{m}, l}^{n_{1}}$.

In the second phase, the relay $l$ decodes and forwards its received signals and the destination $D_{m}$ receives them. Assume that the relayl transmits its received signals with power $p_{l, D_{m}}^{K_{l}}$ over the subcarriers set $K_{l}$, i.e., $K_{l} \subset \mathcal{N},\left|K_{l}\right|>1$. Let $p_{l, D_{m}}^{n_{2}}$ denote the transmit power on the subcarrier $n_{2}$, i.e., $n_{2} \in K_{l}$ and let $p_{l, D_{m}}^{K_{1}}=\sum_{K_{l}} p_{l, D_{m}}^{n_{2}}$. Let $g_{l, D_{m}}^{K_{l}}$ as the channel gain on the subcarriers set $K_{l}$ from the relay $l$ to the destination $D_{m}$ and let $g_{l, D_{m}}^{n_{2}}$ as the channel gain on the subcarrier $n_{2}$. Then let $g_{l, D_{m}}^{K_{1}}=\sum_{K_{l}} g_{l, D_{m}}^{n_{2}}$.

Motivated by the literature [12], a better performance can be achieved if subcarriers in the first and second phases are paired according to the circumstances. We assume that multiple subcarriers are paired for the cooperative communication, while authors in [24] admit only one subcarrier is paired for multi-relay system. For the source-destination pair $\left(S_{m}, D_{m}\right)$, define $\rho_{N_{m}, K_{l}}$ as the identifier, with $\rho_{N_{m}, K_{l}}=1$, which means that $\left(N_{m}, K_{l}\right)$ as the multisubcarriers sets are paired; otherwise, it is zero. Define $a_{m, l}^{\left(N_{m}, K_{l}\right)}$ as the identifier, with $a_{m, l}^{\left(N_{m}, K_{l}\right)}=1$, which means that the relay $l$ is selected and the multi-subcarrier pair is assigned to the source $S_{m}$ and the relay $l$; otherwise, it is zero.

In phase 1 , the throughput of the link from the source $S_{m}$ to relay $l$ can be expressed as

$$
R_{S_{m}}=\left|N_{m}\right|(B / N) \rho_{N_{m}, K_{l}} a_{m, l}^{\left(N_{m}, K_{l}\right)} \log _{2}\left(1+\gamma_{S_{m}, l}^{N_{m}}\right)
$$

where $\gamma_{S_{m}, l}^{N_{m}}$ is the SINR for the source $S_{m}$ using the subcarriers set $N_{m}$. It is given by

$$
\gamma_{S_{m}, l}^{N_{m}}=p_{S_{m}, l}^{N_{m}} g_{S_{m}, l}^{N_{m}} /\left(I_{S_{m}, l}^{N_{m}}+\sigma_{S_{m}}^{2}\right)
$$

where $\sigma_{S_{m}}^{2}$ denotes the additive white Gaussian noise (AWGN) power, and $I_{S_{m}, l}^{N_{m}}$ denotes the interference coming from other sources using the same subcarriers, which is shown in Fig. 1. If $N_{m^{\prime}} \cap N_{m}=\varnothing, m^{\prime} \in\{1,2, \ldots, M\}$, we would set the interference $I_{S_{m}, l}^{N_{m}}$ to zero. Otherwise, the interference $I_{S_{m}, l}^{N_{m}}$ is given by 


$$
I_{S_{m}, l}^{N_{m}}=\sum_{m^{\prime}=1, m^{\prime} \neq m}^{M} p_{S_{m^{\prime}, l}, g_{S^{\prime}}}^{N_{m^{\prime}, l}}
$$

where $p_{S_{m^{\prime}, l^{\prime}}}^{N_{m^{\prime}}}$ and $g_{S_{m^{\prime}, l}}^{N_{m^{\prime}}}$ denote transmit power and the channel gain on the subcarriers set $N_{m^{\prime}}$ allocated the link from the source $S_{m^{\prime}}$ to the relay $l^{\prime}$ and the relay $l$ respectively, and the relay $l^{\prime}, l^{\prime} \in\{1,2, \ldots, L\}$ is different from the relay $l$.

In phase 2, the throughput of the link from the relay $l$ to the destination $D_{m}$ can be expressed as

$$
R_{l}=\left|K_{l}\right|(B / N) \rho_{N_{m}, K_{l}} a_{m, l}^{\left(N_{m}, K_{l}\right)} \log _{2}\left(1+\gamma_{l, D_{m}}^{K_{l}}\right)
$$

where $\gamma_{l, D_{m}}^{K_{l}}$ is the SINR for the relay $l$ using the subcarriers set $K_{l}$. It is given by

$$
\gamma_{l, D_{m}}^{K_{l}}=p_{l, D_{m}}^{K_{l}} g_{l, D_{m}}^{K_{l}} /\left(I_{l, D_{m}}^{K_{l}}+\sigma_{l}^{2}\right)
$$

where $\sigma_{l}^{2}$ denotes AWGN power, and $I_{l, D_{m}}^{K_{1}}$ denotes the interference coming from other relays using the same subcarriers, which is shown in Fig. 1. If $K_{l^{\prime}} \cap K_{l}=\varnothing, l^{\prime} \in\{1,2, \ldots, L\}$, we would set the interference $I_{l, D_{m}}^{K_{l}}$ to zero. Otherwise, the interference $I_{l, D_{m}}^{K_{l}}$ is given by

$$
I_{l, D_{m}}^{K_{1}}=\sum_{l^{\prime}=1, l^{\prime} \neq l}^{L} p_{l^{\prime}, D_{m^{\prime}}}^{K_{K^{\prime}}} g_{l^{\prime}, D_{m}}^{K_{l^{\prime}}}
$$

where $p_{l^{\prime}, D_{m^{\prime}}}^{K_{r}}$ and $g_{l^{\prime}, D_{m}}^{K_{r}}$ denote transmit power and the channel gain on the subcarriers set $K_{l}$, allocated the link from the relay $l^{\prime}$ to the destination $D_{m^{\prime}}$ and the destination $D_{m}$ respectively.

As the throughput of the links from the sources to relays and from relays to the destinations is limited by either the first phase or the second phase, the throughput of the source-destination pair $\left(S_{m}, D_{m}\right)$ can be calculated as

$$
R_{m}=\min \left(R_{S_{m}}, R_{l}\right)
$$

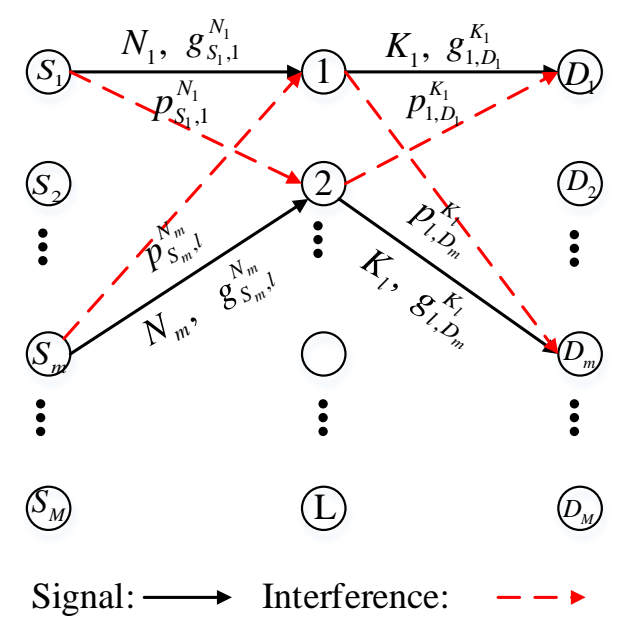

Fig. 1. A relay-aided cooperative system with multiple users and relays 


\subsection{Problem Formulation}

\section{A. MOS Model}

To better consider the practical characteristics of services, QoE is defined basically as a subjective measurement, which contains both QoS parameters and service characteristics [14]. In this paper, we take the delay-tolerate FD (file down) services into consideration when the delay characteristics of relay-aided cooperative system cannot be ignored. Furthermore, we consider the MOS value as the metric of QoE of FD services. Additionally, we assume that all the FD services have the same priority. The logarithmic MOS-throughput model proposed in [25] is used in our work. Here, we assume that the number of the source-destination pairs and the FD services are equal and each source-destination pair transmits one FD service. Thus, the relationship model between MOS and the throughput of each source-destination pair can be described as follows

$$
\operatorname{MOS}_{m}\left(R_{m}\right)= \begin{cases}1, & R_{m} \leq 10 k b p s \\ \alpha \log _{10}\left(\beta R_{m}\right), & 10 k b p s \leq R_{m} \leq 300 k b p s \\ 4.5, & R_{m} \geq 300 k b p s\end{cases}
$$

where $\alpha$ and $\beta$ can be calculated from the upper bound and lower bound of FD service perceived quality , and they are 2.3695 and 0.2643 , respectively.

Then the total MOS value of FD services can be given by

$$
u=\sum_{m=1}^{M} M_{m}
$$

\section{B. Power Consumption Model}

In this paper, the total power consumption of the relay-aided cooperative system contains four terms. The first two terms is the power consumed in the power transmission of the links from the sources to relays and from relays to the destinations. The last two terms is the power used in the circuits of the sources and relays, which are modeled by constants, $P_{c}^{S_{m}}$ and $P_{c}^{l}$ respectively. Then the simple power consumption model of the relay-aided cooperative system is given by

$$
P_{\text {total }}=\sum_{m=1}^{M} \sum_{l=1}^{L} \rho_{N_{m}, K_{l}} a_{m, l}^{\left(N_{m}, K_{l}\right)}\left(p_{S_{m}, l}^{N_{m}}+p_{l, D_{m}}^{K_{l}}+P_{c}^{S_{m}}+P_{c}^{l}\right)
$$

\section{Resource Allocation Model}

The objectives of our resource allocation schemes are to maximize the total MOS value and minimize the total power consumption of the relay-aided cooperative system. Therefore, we formulate resource allocation as a multi-objective optimization problem and the corresponding model is as following

$$
\begin{aligned}
& f_{1}=\max _{\mathbf{p}, \mathbf{a}, \mathbf{p}} u \\
& f_{2}=\max _{\mathbf{p}, \mathbf{a}, \mathbf{p}}-P_{\text {total }} \\
& \text { s.t.: } \\
& \text { C1: } \sum_{m=1}^{M} \rho_{N_{m}, K_{l}} \leq 1, \forall K_{l} \\
& \text { C2: } \sum_{l=1}^{L} \rho_{N_{m}, K_{l}} \leq 1, \forall N_{m}
\end{aligned}
$$




$$
\begin{aligned}
& \text { C3: } \sum_{m=1}^{M} \sum_{l=1}^{L} a_{m, l}^{\left(N_{m}, K_{l}\right)} \leq 1, \forall N_{m}, K_{l} \\
& \text { C4: } \rho_{N_{m}, K_{l}} \in\{0,1\}, a_{m, l}^{\left(N_{m}, K_{l}\right)} \in\{0,1\}, \forall N_{m}, K_{l}, m, l \\
& \text { C5: } 0 \leq p_{S_{m}, l}^{N_{m}} \leq p_{S}^{\max }, 0 \leq p_{l, D_{m}}^{K_{l}} \leq p_{\text {relay }}^{\max }, \forall N_{m}, K_{l}, m, l
\end{aligned}
$$

where $\boldsymbol{\rho}=\left\{\rho_{N_{m}, K_{l}}\right\}, \boldsymbol{\alpha}=\left\{a_{m, l}^{\left(N_{m}, K_{l}\right)}\right\}, \mathbf{p}=\left\{p_{S_{m},}^{N_{m}}, p_{l, D_{m}}^{K_{1}}\right\}, p_{S_{m}}^{\max }$ and $p_{l}^{\max }$ are the maximum transmit power of the sources and relays respectively. Constraints C1 and C2 ensure that each subcarriers set is only paired with one subcarriers set in each link. Furthermore, constraint C3 enforces assignment of one multi-subcarrier pair $\left(N_{m}, K_{l}\right)$ to only one source and relay. Constraint C4 ensures that the indicators of the subcarriers paired and the relay selected are binary variables. Where $\mathrm{C} 5$ is the power constraints of the sources and relays.

\section{Resource Allocation Based on Multi-Objective Optimization}

\subsection{Multi-Objective Optimization Algorithm}

The traditional methods used for solving multi-objective optimization problems can be decomposed into two broad groups: gradient-based and genetic methods based [26]. The main contribution of the genetic algorithm over the gradient-based method is that it eliminates the computation of derivatives of the objective functions and constraint equations. NSGA is a popular non-domination based genetic algorithm for multi-objective optimization. NSGA-II [27], as a modified version of NSGA, which has been generally criticized for its computational complexity and lack of elitism, is developed. The general description of NSGA-II is as following. Firstly, the population is initialized as usual. Once the population in initialized the population is sorted based on non-domination into each front. Individuals in first front are given a fitness (rank) value of 1 and individuals in second are assigned fitness value as 2 and so on. Secondly, in addition to fitness value a new parameter called crowding distance is caculated for each individual. Large average crowding distance will result in better diversity in the population. Thirdly, parents are selected from the population by using tournament selection based on the rank and crowding distance. An individual is selected in the rank is lesser than the other or if crowding distance is greater than the other. The selected population generates offsprings from crossover and mutation operators. Finally, the population with the current population and current offsprings is sorted again based on non-domination and only the best $\mathcal{X}$ individuals are selected, where $\mathcal{X}$ is the population size. The selection is based on rank and the crowding distance on the last front. Algorithm 1 displays the concrete description of NSGA-II.

Similar to the literature [28], NSGA-II, as an effective genetic algorithm, is used to solve multi-objective optimization problems and to obtain the set of Pareto optimal solutions, where we find the optimal solutions of the multi-objective optimization problems with and without a service priority constraint. Then we obtain the optimal resource allocation schemes for maximizing the total MOS value and minimizing the total power consumption of the relay-aided cooperative system.

Let the optimizing variables $\rho_{N_{m}, K_{l}}, a_{m, l}^{\left(N_{m}, K_{l}\right)}, p_{S_{m}, l}^{N_{m}}$ and $p_{l, D_{m}}^{K_{l}}$ be a multigene, which is the extended from one gene in [28] . Let the indicators of the subcarriers paired $\rho$, the relay selected and the multi-subcarrier pair assigned $\boldsymbol{\alpha}$, and the transmit power of the sources and relays $\mathbf{p}$ be a resource allocation matrix $\mathbf{H}=[\boldsymbol{p}, \boldsymbol{\alpha}, \mathbf{p}]$. Furthermore, let the matrix $\mathbf{H}$ be an 
individual. A population is composed of multiple allocation matrixes $\mathbf{H}$. First, A population is initialized with a multigene pool consisting of many individuals and a non-dominant operator for quick sorting of this population is implemented. Second, we use rank-based model in consideration of the non-dominant sorting to keep the diversity of the population. Finally, we employ the elite strategy to ensure the great individuals into the child directly which is to prevent the Pareto optimal solutions from missing. The process of the pseudo-code for resource allocation through the NSGA-II is proposed as follows.

Algorithm 1 Multi-objective Optimization Algorithm

- Initialization:

- Set $g=0$ and the length of each multigene is $M$. So each multigene matrix $\mathbf{H}=[\boldsymbol{\rho}, \boldsymbol{\alpha}, \mathbf{p}]_{4 \times M}$ represents a resource allocation scheme.

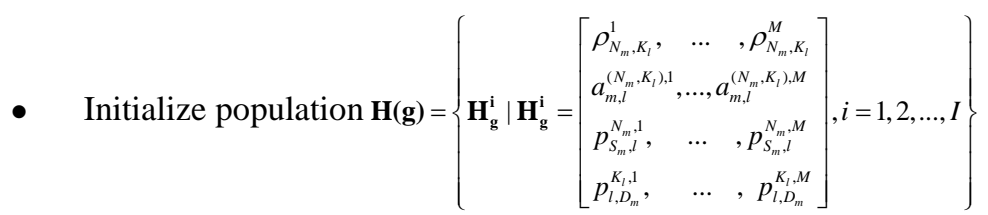

Corresponding to the size of $I$. Randomly select multigene as an individual $\mathbf{H}_{\mathrm{g}}^{\mathrm{i}}$ and substitute $\mathbf{H}_{\mathrm{g}}^{\mathrm{i}}$ in the constraint (C1)-(C5). If the constraint (C1)-(C5) is not satisfied, we would repeat it till total number of $\mathbf{H}_{\mathrm{g}}^{\mathrm{i}}$ being $I$.

- Repeat:

- $\quad$ Sorting: Sort individuals in $\mathbf{H ( g )}$ and get new child population $\mathbf{H}^{1} \mathbf{( g )}$ using

(1) Non-dominated sort.

(2) Crowding distance.

- Selection: Select individuals from $\mathbf{H}^{1}(\mathbf{g})$ in order to fill the mating pool employing tournament selection and crowding selection operator.

- Crossover: With a crossover probability cross over the parents to form new offspring (children). If no crossover is performed, offspring will be the exact copy of parents.

- Mutation: With a mutation probability mutate new offspring at each locus (position in chromosome).

- Accepting: Place new offspring in $\mathbf{H}^{2}(\mathbf{g})$. And merge parent generation and child generation $\mathbf{H}^{3}(\mathbf{g})=\mathbf{H}^{1}(\mathbf{g}) \cup \mathbf{H}^{2}(\mathbf{g})$.

- $\quad$ Replace: Copy new generated population $\mathbf{H}^{3}(\mathbf{g})$ to $\mathbf{H}(\mathbf{g})$ and Set $g=g+1$.

- Test and loop:

- If stopping, criterion are satisfied (e.g. reaches a constant number of generations in this paper) and the best solution in current population is returned. Otherwise go to step 4(Repeat).

\subsection{Choice of the Optimal Solution without a Service Priority Constraint}

The optimal value of the problem can be obtained from a set of Pareto-optimal solution. Our purpose is to improve the total MOS value and reduce the total power consumption of the system. We employ a formula (13), which is devised in our previous publication [28].

$$
\arg \max \left\{\frac{f_{1 j}}{f_{1 \max }}+\frac{f_{2 j}}{f_{2 \max }}\right\}, j=1,2, \ldots, J
$$

The maximum of formula (13) is solved and then optimal values of $f_{1}$ and $f_{2}$ are selected, which is also consistent with solving the optimal solution of multi-objective problem in this paper. 


\subsection{Choice of the Optimal Solution with a Service Priority Constraint}

In the above discussions, we assume that all the FD services have the same priority. This assumption simplifies the discussion for practical systems. However, in the practical systems, not all FD services have the same the priority. Therefore, in this section, we assume that the number of the source-destination pairs and the FD services are equal to . FD services are sorted by priority, which can ensure that the smaller rank is, the higher priority of the FD service has. We also design a formula as is shown below.

$$
\left\{\begin{array}{l}
\arg \max \left\{\frac{f_{1 j}}{f_{1 \max }}+\frac{f_{2 j}}{f_{2 \max }}\right\}(14 a), \text { if } m=1 \\
\arg \min \left\{\sum_{m}\left(f_{1, j^{\text {prority }}(m-1)}^{m-1}-f_{1, j}^{m-1}\right)\right\}(14 b), \text { if } m>1
\end{array}\right.
$$

where $j=1,2, \ldots, J$. Let $J$ denotes the number of Pareto-optimal solution. If $m=1$, the maximum of formula (14a) is solved as same as (13). Otherwise, we should solve the

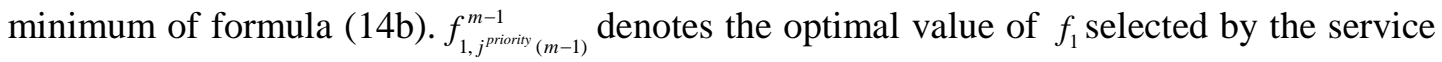
with the rank $m-1$. $\arg \min \left\{\sum_{m}\left(f_{1, j \text { proriry }(m-1)}^{m-1}-f_{1, j}^{m-1}\right)\right\}$ means the optimal Pareto-optimal solution $j$ having minimum effect on the values of $f_{1}$ for services with the ranks from $m-1$ to 1 , is selected by the service with the rank $m$. Then the optimal values of $f_{1}$ and $f_{2}$ are selected. That means the optimal solution of multi-objective problem with a service priority constraint is obtained.

\section{Simulation Results and Discussions}

Here, we compare the performance of the proposed resource allocation schemes based on the NSGA-II with existing ones. In our simulation, the resource allocation scheme proposed in [12] is called the existing scheme 1 with the only one subcarrier paired in the multi-relay system. Furthermore, we add a service priority condition to the existing scheme 1 and call it the existing scheme 2. Additionally, the resource allocation scheme proposed in [10] is called the existing scheme 3 with the only one subcarrier paired in the single relay system. One of the proposed resource allocation schemes is called the proposed scheme 1 using the NSGA-II to solve the resource allocation with multiple subcarriers paired in the multi-relay system. The other is called the proposed scheme 2 where we add a service priority condition to the proposed scheme 1.

All the experiments are performed in the same simulation scenarios as shown in Fig. 1. Here, $p_{s_{m}}^{\max }$ as the maximum transmit power of the sources and $p_{l}^{\max }$ as the maximum transmit power of the relays are equal, and they are denoted by $p^{\max }$. We assume that the distance between each source and destination is $500 \mathrm{~m}$, and the relays are located between the sources and the destinations randomly. The detail description of the simulation parameters used in our experiments is display in Table 1.

The effectiveness of any evolutionary algorithm depends on the choice of its parameters. Selections of the better parameters of the NSGA-II are shown as follows [29]: $L=150$, gen $=100, p c=0.9, p m=0.03$. Where $L$ is the population size fixed a priori to 150 , gen is the maximum number of generations, $p c$ is the crossover probability, $p m$ is the mutation probability. 
Table 1. Simulation Parameters

\begin{tabular}{|c|c|}
\hline Parameter & Value \\
\hline \hline Total bandwidth $(B)$ & $500 \mathrm{KHz}$ \\
\hline Number of subcarriers $(N)$ & 10 \\
\hline The AWGN power spectral density $(\mathrm{NO})$ & $-136 \mathrm{dBm}$ \\
\hline Constant power consumption of the sources $\left(P_{c}^{S_{m}}\right)$ & $500 \mathrm{~mW}$ \\
\hline Constant power consumption of the relays $\left(P_{c}^{l}\right)$ & $1000 \mathrm{~mW}$ \\
\hline The exponent of large-scale fading $(\alpha)$ & 3.5 \\
\hline
\end{tabular}

Fig. 2 shows the sets of Pareto optimal solutions both in proposed scheme 1 and 2, where $M=2, p^{\max }=1 W$. As depicted in Fig. 2 (a), we choose a optimal solution for our multi-objective problem from the Pareto front based on formula (13), which is marked in red. Clearly, two Pareto fronts are shown in Fig. 2 (b). Becauese the two services with a different priority rank are taken into consideration, we choose a optimal solution of our multi-objective problem with a service priority cinstraint from the first Pareto front based on formula (14a) for the higher priority service with the rank 1 and then choose a optimal solution from the second Pareto front based on formula (14b) for the lower priority service with the rank 2, which are also marked in red. Similar to Fig. 2, other sets of Pareto optimal solutions can be also obtained both in proposed scheme 1 and 2 with other simulation parameters, i.e., $M=3$, and corresponding optimal solution is shown as follows.

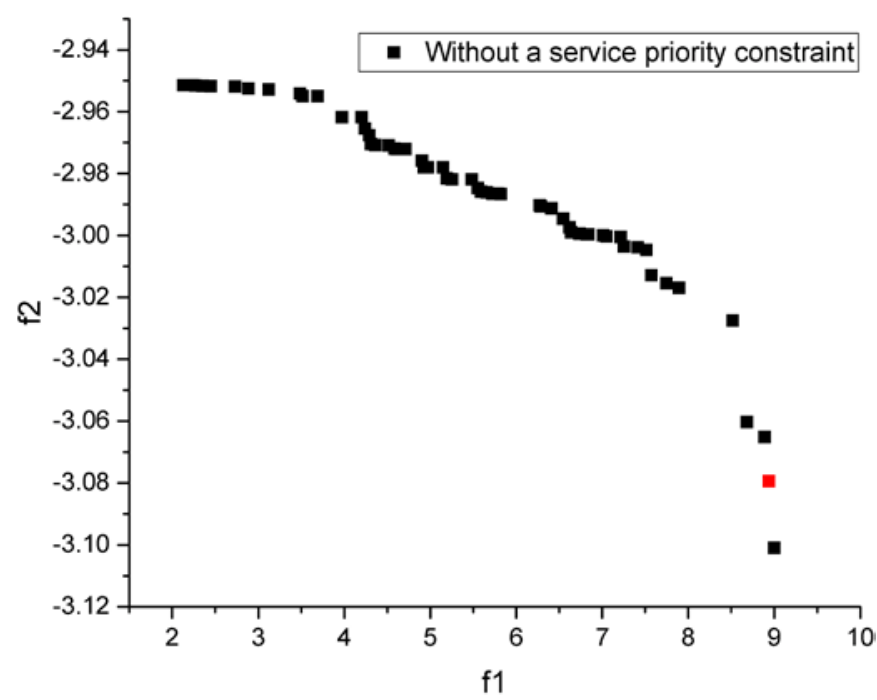

(a) The proposed scheme 1 


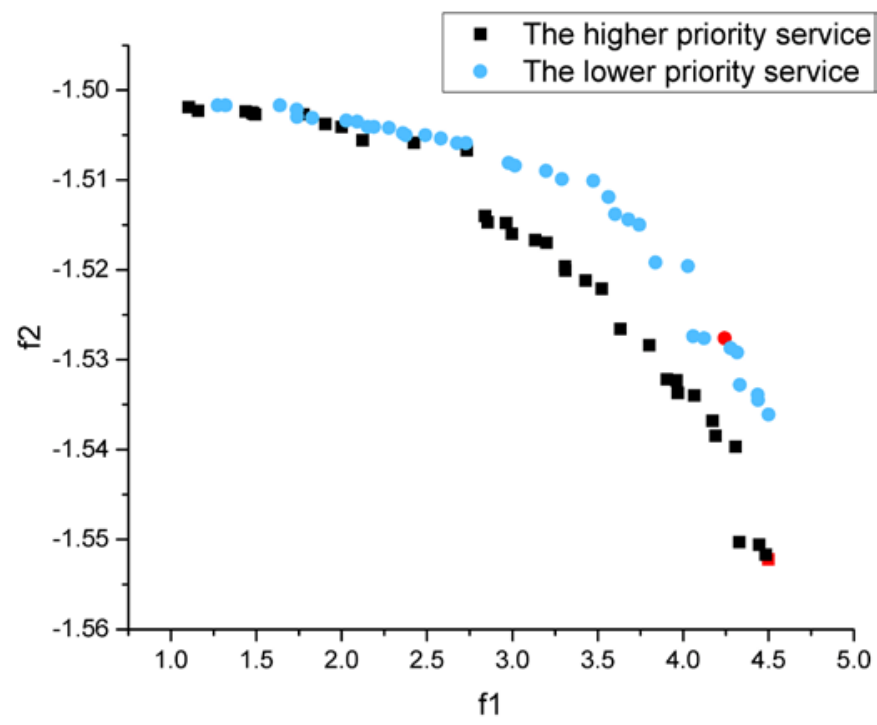

(b) The proposed scheme 2

Fig. 2. The sets of Pareto optimal solutions

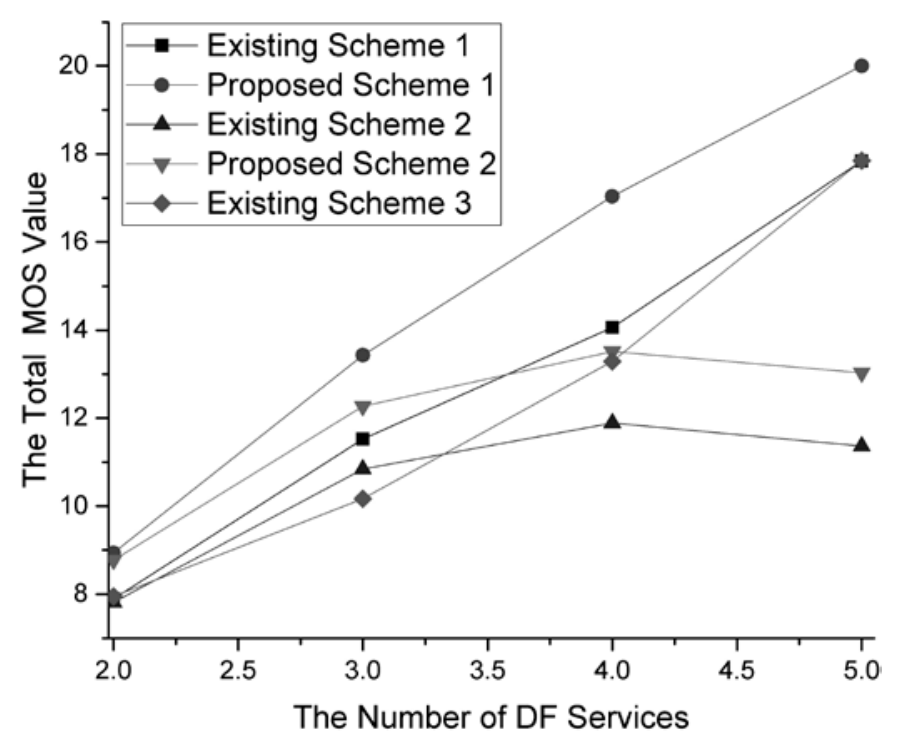

Fig. 3. The total MOS value versus the number of service

Fig. 3 shows the total MOS value when the number of DF services increases from 2 to 5 both in proposed schemes and the existing schemes. The simulation parameter is set as $p^{\max }=1 \mathrm{~W}$. As depicted in Fig. 3, the proposed scheme 1 always provides the higher MOS value when compared with the existing scheme 1 and 3 . The proposed scheme 2 always provides the higher MOS value when compared with the existing scheme 2 . This is because that the existing scheme 1, 2and 3 admit that one subcarrier is paired and equal subcarriers are assigned in each phase to each source-destination pair, which limits the QoE of services. Additionally, there is only one relay in the existing scheme 3 , thus the relay selection is ignored. This limits the flexibility of resource allocation, and cannot ensure QoE of all the services at the same time. 
As the number of the subcarriers in each phase is fixed as $N$, with the increase of the number of services, each subcarrier has more candidate sources to select, which is known as multiuser diversity. Thus, as the number of DF services rises, the total MOS value increases. However, the proposed scheme 2 and the existing scheme 2 vary with the number of services increasing firstly and then decreasing. This is due to the services priority case. In this case, QoE of the higher priority services firstly ensured. Note that in (14b), choice of the optimal solution enforces the lower priority services choosing the resource allocation scheme that has a minimum effect on QoE of the higher priority services. This cannot ensure QoE of the lower priority services when the resource is fixed.

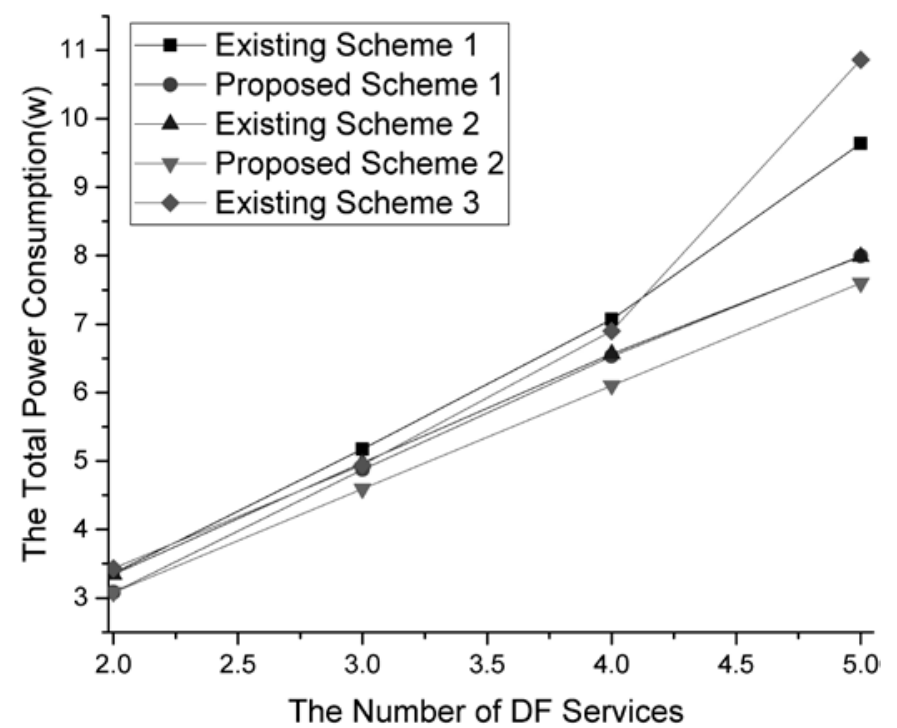

Fig. 4. The total power consumption versus the number of services

Fig. 4 shows the total power consumption when the number of DF services increases from 2 to 5 both in proposed schemes and the existing schemes. The simulation parameter is also set as $p^{\max }=1 W$. As depicted in Fig. 4, the proposed scheme 1 and 2 always provides the lower total power consumption when compared with the existing scheme 1, 2 and 3 . This is because that the existing schemes admit that only one subcarrier is paired and equal subcarriers are assigned in each phase to each source-destination pair. This requires more transmit power for providing the higher MOS value than the proposed schemes where multiple subcarriers are paired and unequal subcarriers are assigned in each phase to each source-destination pair. Additionally, there is only one relay in the existing scheme 3 , thus the increasing trend varies with the number of DF services increasing for providing the higher MOS value of all services.

To quantify the performance between MOS value and power consumption, the metric of MOS value per Joule is defined as $u / P_{\text {total }}$, which is similar to the metric of EE. The performance of the defined metric is shown in Fig. 5 -6.

Fig. 5 shows MOS value per Joule when the number of DF services increases from 2 to 5 both in proposed schemes and the existing schemes. The simulation parameter is also set as $p^{\max }=1 W$. As depicted in Fig. 5, the proposed scheme 1 outperforms the existing scheme 1 and 3 . The proposed scheme 2 also outperforms the existing scheme 2 in terms of the defined 
metric with the increase in number of services from 2 to 5 . However, both the proposed schemes and the existing schemes have the same trends with the increase in number of services from 2 to 5 . With the increase in the index number of DF services, the MOS value per Joule decrease. This is due to the interference in the proposed schemes will increase with the increase in number of services from 2 to 5, which can impair the potential gain of the total MOS value $u$. Additionally, as depicted in Fig. 4, the total power consumption $P_{\text {total }}$ in the existing schemes will increase significantly, which leads to the decrease of the MOS value per Joule.

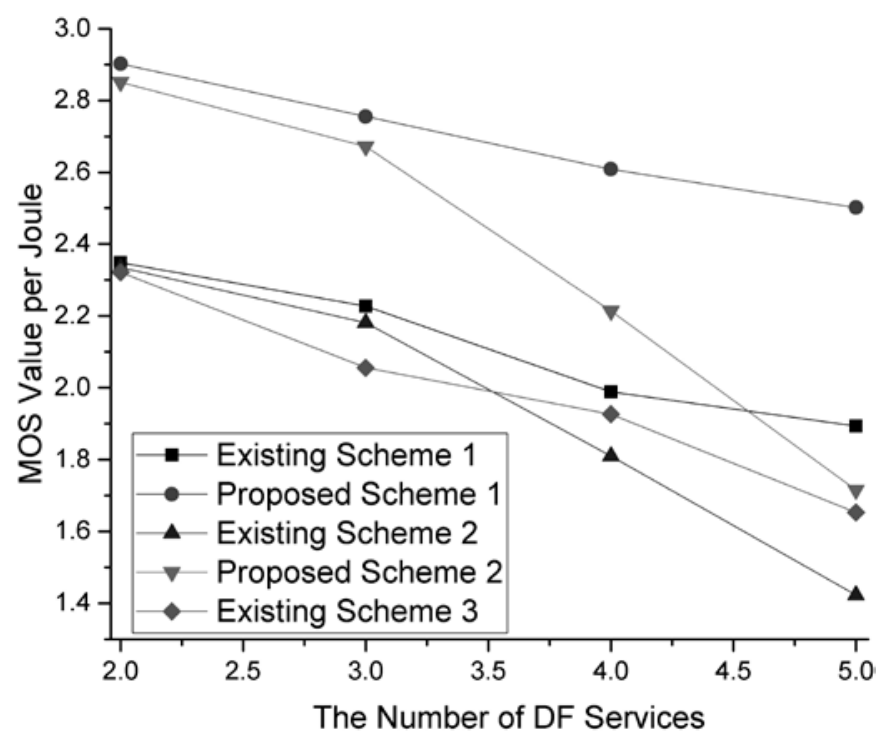

Fig. 5. MOS value per Joule versus the number of services

Fig. 6 shows MOS value per Joule when the power $p^{\max }$ increases from 0.2 to $1 \mathrm{~W}$ both in proposed schemes and the existing schemes. The simulation parameter is set as $M=2$. We can see that the proposed schemes also outperform the existing schemes. With the increase in the power $p^{\max }$, the MOS value per Joule increases for both the proposed schemes and the existing schemes. However, the increasing trend varies with the power decreasing because of the defined metric, which is in inverse proportion to the total power consumption.

Fig. 7 shows that the convergence in terms of the power consumption for the NSGA-II versus the number of generations, where $M=2, p^{\max }=1 W$. It can be observed that the NSGA-II takes tens generations to converge to stable solutions. This result ensures the proposed schemes are suitable for practical wireless applications. 


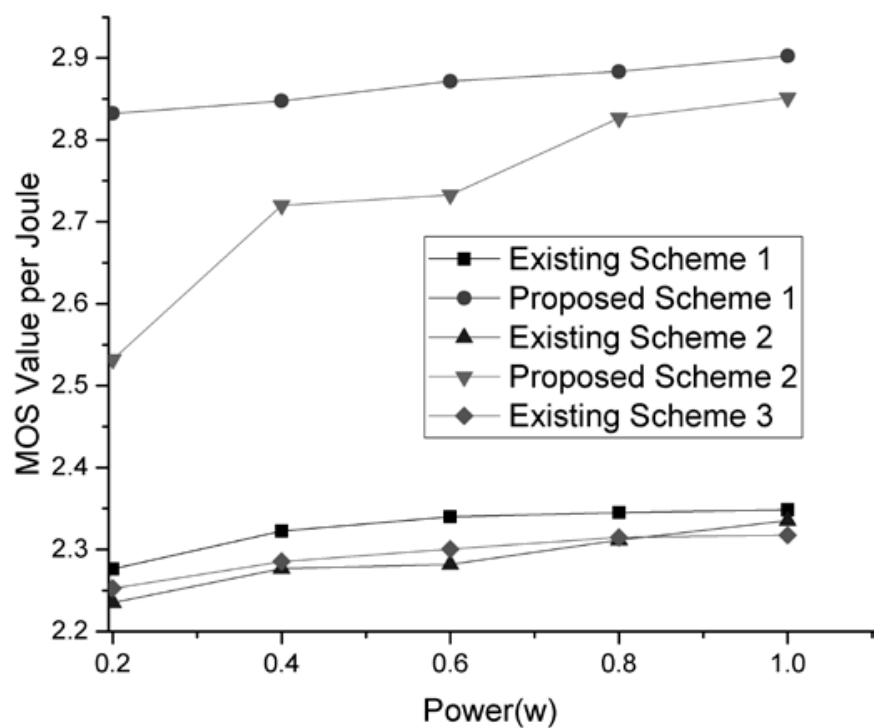

Fig. 6. MOS value per Joule versus different maximum power

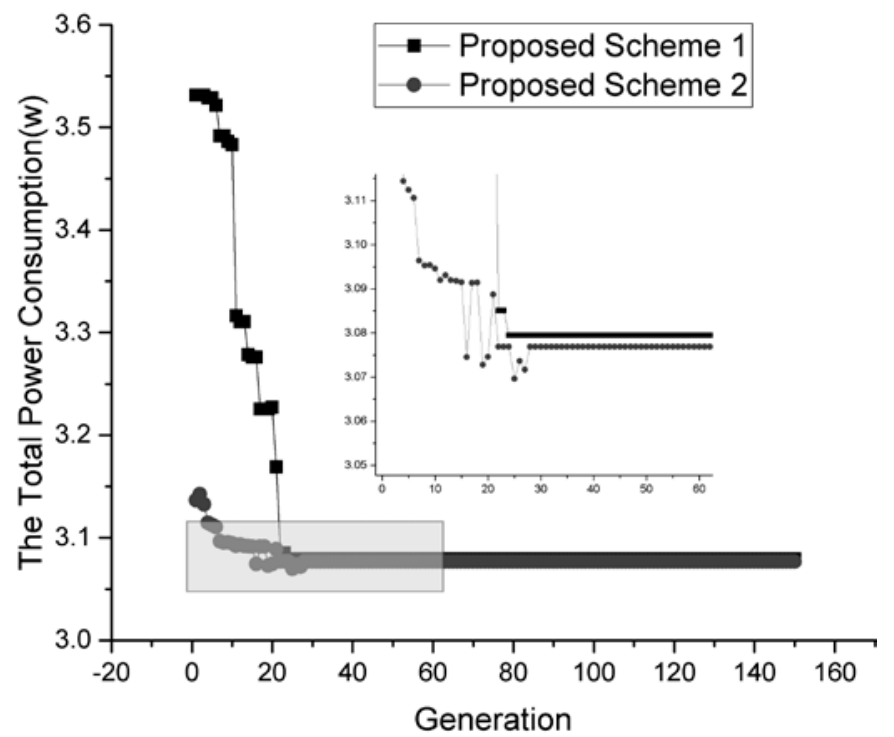

Fig. 7. The convergence in terms of the total power consumption versus the number of iterations

\section{Conclusion}

In this paper, two resource allocation schemes based on multi-objective optimization have been proposed. The implementation of NSGA-II was then introduced to solve the multi-objective optimization problem. Simulation results indicated that the proposed schemes offer significantly higher MOS value and lower power consumption than the existing schemes. Specifically, the results of the metric of MOS value per Joule obtained by the proposed schemes are also higher than those obtained by the existing schemes. At last, our methods demonstrate that the proposed schemes efficiently manage the resource allocation between source-destination pairs and relays. 


\section{References}

[1] Li Q, Yu M, Pandharipande A, et al., "Performance of Virtual Full-Duplex Relaying on Cooperative Multi-Path Relay Channels," IEEE Transactions on Wireless Communications, vol.15, no.8, pp. 3628-3642, 2016. Article (CrossRef Link)

[2] Li Q, Yu M, Pandharipande A, et al., "Outage Analysis of Co-Operative Two-Path Relay Channels,” IEEE Transactions on Wireless Communications, vol.15, no.5, pp. 3157-3169, 2016. Article (CrossRef Link)

[3] C. Hoymann, W. Chen, J. Montojo, and A. Golitschek, "Relaying operation in 3GPP LTE: challenges and solutions,” Communications Magazine IEEE, vol.50, no.2, pp. 156-162, 2012. Article (CrossRef Link)

[4] Nosratinia, A, T. E. Hunter, and A. Hedayat, "Cooperative communication in wireless networks," Communications Magazine IEEE, vol.42, no.10, pp. 74-80, 2004. Article (CrossRef Link)

[5] Gao H, Zhang S, Du Y, et al., "Relay selection scheme based on quantum differential evolution algorithm in relay networks,” KSII Transactions on Internet \& Information Systems, vol.11, no.7, pp. 3501-3523, 2017.Article (CrossRef Link)

[6] Liang, Liang, G. Feng, and Y. Jia, "Game-Theoretic Hierarchical Resource Allocation for Heterogeneous Relay Networks,” IEEE Transactions on Vehicular Technology, vol.64, no.4, pp. 1480-1492, 2015. Article (CrossRef Link)

[7] C. Z. Chen, T. Li, P. Y. Fan, et al., "Cooperation in 5G Heterogeneous Networking: Relay Scheme Combination and Resource Allocation,”IEEE Transactions on Communications, vol.64, no.8, pp. 3430-3443, 2016. Article (CrossRef Link)

[8] T. T. Yu, Y. L. Jin, W. S. Guo, et al., "Low-complexity energy-efficient resource allocation for delay-tolerant two-way orthogonal frequency-division multiplexing relays,” Iet Communications, vol.10, no.17, pp. 2488-2495, 2016. Article (CrossRef Link)

[9] S. Kim and H. Yu, "Energy-efficient resource allocation in multi-user AF two-way relay channels,” Journal of Communications \& Networks, vol.18, no.4, pp. 629-638, 2016.

Article (CrossRef Link)

[10] S. G. Wang, R. Ruby, Z.Q. Yao, “A Low-Complexity Power Allocation Strategy to Minimize Sum-Source-Power for Multi-User Single-AF-Relay Networks," IEEE Transactions on Communications, vol.64, no.8, pp. 3275-3283, 2016. Article (CrossRef Link)

[11] Z. Y. Song, Q. Ni , K. Navaie, and S.J. Hou, et al., “On the Spectral-Energy Efficiency and Rate Fairness Tradeoff in Relay-Aided Cooperative OFDMA Systems,'IEEE Transactions on Communications, vol.15, no.9, pp. 6342-6355, 2016. Article (CrossRef Link)

[12] W. Li, J. Lei, T. Wang, et al., "Dynamic Optimization for Resource Allocation in Relay-Aided OFDMA Systems Under Multiservice,” IEEE Transactions on Vehicular Technology, vol.65, no.3, pp. 1303-1313, 2016. Article (CrossRef Link)

[13] G. Liu, F. Richard, H. Ji, et al., "Distributed Resource Allocation in Virtualized Full-Duplex Relaying Networks,” IEEE Transactions on Vehicular Technology, vol.65, no.10, pp. 8444-8460, 2016. Article (CrossRef Link)

[14] N. W. Wang, Z. S. Fei, and J. M. Kuang, “ QoE-Aware Resource Allocation for Mixed Traffics in Heterogeneous Networks Based on Kuhn-Munkres Algorithm,” in Proc. of 2016 IEEE International Conference on Communication Systems (ICCS), Dec. 2016. Article (CrossRef Link)

[15] Z. S. Fei, C. W. Xing, and N. Li, “QoE-driven resource allocation for mobile IP services in wireless network,” Science China Information Sciences, vol.58, no.1, pp. 1-10, 2015. Article (CrossRef Link)

[16] J. Y. Dai, and S. Wang, "QoE-driven resource allocation method for cognitive radio networks," in Proc. of IEEE International Conference on Communications, May 2016. Article (CrossRef Link)

[17] S. Y. Zhou, M. Ran, and Z. Lu, “Adaptive energy-efficient and QoE-aware optimization method for mobile video services,” in Proc. of 2016 16th International Symposium on Communications and Information Technologies (ISCIT), Sept. 2016. Article (CrossRef Link) 
[18] N. Zhang, S. Zhang, J. Zheng, et al., "QoE Driven Decentralized Spectrum Sharing in 5G Networks: Potential Game Approach,” IEEE Transactions on Vehicular Technology, vol. PP, no.99, pp. 1-6, 2017. Article (CrossRef Link)

[19] T. Hossfeld, L. Skorin-Kapov, P. Heegaard ,et al., "Definition of QoE Fairness in Shared Systems," IEEE Communications Letters, vol.21, no.1, pp. 184-187, 2017. Article (CrossRef Link)

[20] D. C. Wu, Q. H. Wu, Y. H. Xu, et al., “QoE and Energy Aware Resource Allocation in Small Cell Networks with Power Selection, Load Management and Channel Allocation,” IEEE Transactions on Vehicular Technology, vol.PP, no.99, pp. 1-13, 2017. Article (CrossRef Link)

[21] D.C.Wu, Q. Wu, Y. XU, et al., "QoE-Based Distributed Multichannel Allocation in 5G Heterogeneous Cellular Networks: A Matching-Coalitional Game Solution,” IEEE Access, no.99, pp. 61-71, 2016. Article (CrossRef Link)

[22] Lin, Chun Cheng, L. Shu, and D. J. Deng. "Router Node Placement With Service Priority in Wireless Mesh Networks Using Simulated Annealing With Momentum Terms,” IEEE Systems Journal, vol. PP, no.99, pp. 1-10, 2016. Article (CrossRef Link)

[23] Zhang, Deng Yin, L. Cui, and Z. Y. Tong. "A Handoff Control Algorithm Based on Services-Priority and Channel Reservation,” in Proc. of International Conference on Information Technology, Computer Engineering and Management Sciences IEEE, Dec.2011. Article (CrossRef Link)

[24] W. B. Dang, M. X. Tao, H. Mu, et al., "Subcarrier-Pair Based Resource Allocation for Cooperative AF Multi-Relay OFDM Systems,” IEEE Transactions on Wireless Communications, vol.9, no.5, pp. 640-1649, 2010. Article (CrossRef Link)

[25] F. Kelly, "Charging and rate control for elastic traffic," Transactions on Emerging Telecommunications Technologies, vol.8, no.1, pp. 33-37, 1997. Article (CrossRef Link)

[26] H. Zhang, C. Jiang, and N. C. Beaulieu, "Resource Allocation for Cognitive Small Cell Networks: A Cooperative Bargaining Game Theoretic Approach,” IEEE Transactions on Wireless Communications, vol. 65, no. 3,pp. 3481-3493, 2015. Article (CrossRef Link)

[27] Deb K, Pratap A, Agarwal S, et al., "A fast and elitist multiobjective genetic algorithm: NSGA-II,” IEEE Transactions on Evolutionary Computation, vol. 6, no.2, pp.182-197, 2002. Article (CrossRef Link)

[28] R. Wu, J. Zhu, L. Tang, et al., “A spectrum-sharing Approach in Heterogeneous Networks Based on Multi-Objective Optimization,” IEICE Transactions on Communications, vol. E100.B, no. 7, pp. 1145-1151, July 2017. Article (CrossRef Link)

[29] N. Sharma, D. Badheka, and A. Anpalagan, "Multiobjective Subchannel and Power Allocation in Interference-Limited Two-Tier OFDMA Femtocell Networks,” IEEE Systems Journal, vol. 1, no. 4, pp. 1-12, June 2016. Article (CrossRef Link) 


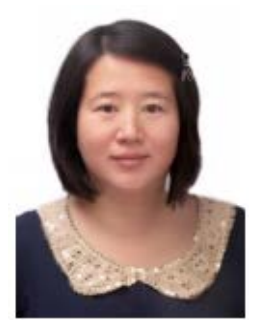

Runze Wu received the Ph.D. degree in Electromagnetic Theory \&Microwave Engineering from Beijing Univerdity of Posts and Telecommunications. Since 2001, she has been an Associate Professor in North China Electric Power University. She is the director of Nation Nature Science Foundation of China (No.51507063). Her main research interests include the new communication technology and its application in Smart Grid.

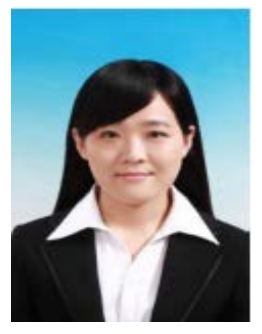

Jiajia Zhu received the B.Sc. degree in Electronic Information Engineering from North China Electric Power University in July 2014. She is currently pursuing her Ph.D. degree in Electrical Engineering of North China Electric Power University. Her research interests cover cognitive radio networks, power wireless communication.

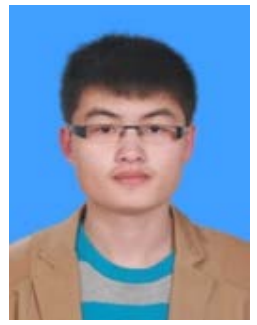

Hailin Hu received the B.Eng. degree in Telecommunications Engineering from North China Electric Power University in July 2016. He is currently pursuing his Ph.D. degree in Electrical Engineering of North China Electric Power University. His research interests cover resource allocation in heterogeneous networks.

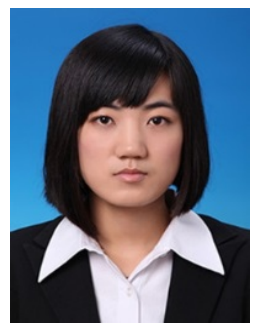

Yanhua He received her B.Eng. (2015) in communication engineering from North China Electric Power University (NCEPU) and she is now pursing her Ph.D. master degree at NCEPU. Her research interests cover interference management and resource allocation in heterogeneous networks.

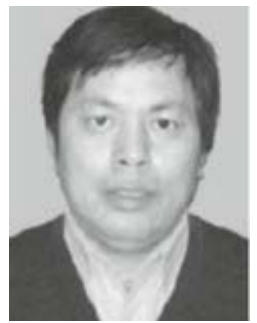

Liangrui Tang received the Ph.D. degree in Communication and Information System from Beijing University of Posts and Telecommunicatons. Now, he is a Professor in State Key Laboratory of Alternate Electrical Power System with Renewable Energy Sources of North China Electric Power University, focusing on the research of communication in power system, wireless communications and optical network communication. 